\title{
ON A THEOREM OF POKORNYI
}

W. J. KIM

Let $p_{0}, p_{1}, \cdots, p_{n-1}$ be analytic functions defined in a region $R$. The differential equation

$$
y^{(n)}+p_{n-1} y^{(n-1)}+\cdots+p_{0} y=0
$$

is said to be disconjugate in $R$ if no nontrivial solution of (1) has more than $n-1$ zeros (where the zeros are counted with their multiplicities) in $R$. For the even-order equation $(n=2 m)$, we may consider a weaker notion of disconjugacy: Equation (1) is said to be disconjugate in the sense of Reid [9] in $R$ if no nontrivial solution of (1) has two zeros of order $m$ in $R$.

Disconjugacy of the second-order equation

$$
y^{\prime \prime}+p y=0
$$

has been studied by Nehari [5], [7], Pokornyi [8], and London [4]; the results are usually formulated as univalence criteria for an analytic function. In [8], Pokornyi announced the following theorem: Let $p$ be analytic in $D=\{z:|z|<1\}$. If

$$
|p(z)| \leqq 2 /\left(1-|z|^{2}\right), \quad z \in D,
$$

then Equation (2) is disconjugate in $D$.

The principal aim of this note is to establish an analogous result for the equation

$$
y^{(2 m)}+p y=0 \text {. }
$$

Theorem I. Let $p$ be analytic in $D=\{z:|z|<1\}$. If

$$
|p(z)| \leqq(2 m) ! /\left(1-|z|^{2}\right)^{m}, \quad z \in D,
$$

then Equation (3) is disconjugate in the sense of Reid in D.

For the proof of the above theorem, we require the following lemma.

Lemma I. Let $y$ be analytic in a region $R$. If $y\left(a_{i}\right)=0, a_{i} \in R$, $i=1,2, \cdots, n$, then

Received by the editors April 11, 1969. 


$$
\begin{aligned}
y\left(z_{0}\right)= & \left(a_{n}-z_{0}\right) \cdots\left(a_{2}-z_{0}\right) \int_{a_{1}}^{z_{0}} \frac{1}{\left(a_{2}-z_{1}\right)^{2}} \int_{a_{2}}^{z_{1}} \frac{a_{2}-z_{2}}{\left(a_{3}-z_{2}\right)^{3}} \cdots \\
& \cdot \int_{a_{n-1}}^{z_{n-2}} \frac{\left(a_{n-1}-z_{n-1}\right)^{n-2}}{\left(a_{n}-z_{n-1}\right)^{n}} \int_{a_{n}}^{z_{n-1}}\left(a_{n}-z_{n}\right)^{n-1} y^{(n)}\left(z_{n}\right) d z_{n} \cdots d z_{1},
\end{aligned}
$$

where the integrations are performed along any curve in $R$ connecting the two points $a_{k}$ and $z_{k-1}, \mathrm{k}=1,2, \cdots, n$ (cf. [1]).

Proof. If $f$ is analytic in $R$ and $f(a)=0, a \in R$, then it is easily confirmed that

$$
\left(\frac{f}{a-z}\right)^{(k)}=\frac{1}{(a-z)^{k+1}} \int_{a}^{z}(a-w)^{k} f^{(k+1)}(w) d w
$$

$k=0,1,2, \cdots$ The lemma now follows from (4) and induction.

Proof of Theorem I. Suppose that Equation (3) has a nontrivial solution $y$ with two zeros $z=a_{1}$ and $z=a_{2}$ of order $m$ in $D$. Choose constants $K$ and $\alpha,|K|=1,|\alpha|<1$, such that the transformation $z=K(w-\alpha) /(1-\bar{\alpha} w)$ carries $z=a_{1}$ and $z=a_{2}$ onto $w=0$ and $w$ $=-\rho, 0<\rho<1$, respectively. Then the function $Y$ defined by

$$
Y(w)=y\left(\frac{K(w-\alpha)}{1-\bar{\alpha} w}\right) \cdot \exp \left[-(2 m-1) \int \frac{\bar{\alpha}}{1-\bar{\alpha} w} d w\right]
$$

has two zeros $w=0$ and $w=-\rho$ of order $m$ and satisfies the differential equation

$$
Y^{(2 m)}+\left[\frac{K\left(1-|\alpha|^{2}\right)}{(1-\bar{\alpha} w)^{2}}\right]^{2 m} q Y=0,
$$

where $q(w)=p(K(w-\alpha) /(1-\bar{\alpha} w))$ (see, e.g., [3]). Furthermore, we have

$$
\left|\frac{K\left(1-|\alpha|^{2}\right)}{(1-\bar{\alpha} w)^{2}}\right|^{2 m}|q(w)| \leqq \frac{(2 m) !}{\left(1-|w|^{2}\right)^{m}}
$$

for $-1<w \leqq 0$. Since the transformation $z=K(w-\alpha) /(1-\bar{\alpha} w)$ can be built up from two rotations and a transformation of the type $z=(w-\beta) /(1-\beta w), 0<\beta<1$, it suffices to establish (6) for these two types of transformations. That (6) holds for $z=K w,|K|=1$, is readily seen. For $z=(w-\beta) /(1-\beta w), 0<\beta<1$, we have 


$$
\begin{aligned}
\left|\frac{1-\beta^{2}}{(1-\beta w)^{2}}\right|^{2 m} \mid p & \left(\frac{w-\beta}{1-\beta w}\right) \mid \\
& \leqq\left|\frac{1-\beta^{2}}{(1-\beta w)^{2}}\right|^{2 m} \frac{(2 m) !}{\left(1-|(w-\beta) /(1-\beta w)|^{2}\right)^{m}} \\
& =\frac{(2 m) !}{\left(1-|w|^{2}\right)^{m}}\left|\frac{1-\beta^{2}}{(1-\beta w)^{2}}\right|^{m} \\
& \leqq \frac{(2 m) !}{\left(1-|w|^{2}\right)^{m}}, \quad-1<w \leqq 0 .
\end{aligned}
$$

We now use Lemma $I$ to express the function $Y$ in the interval $[-\rho, 0]$ :

$$
\begin{aligned}
Y(w)= & -(\rho+w)^{m-1} w^{m} \int_{-\rho}^{w} \frac{1}{\left(\rho+w_{1}\right)^{2}} \int_{-\rho}^{w_{1}} \frac{1}{\left(\rho+w_{2}\right)^{2}} \cdots \\
& \cdot \int_{-\rho}^{w_{m-2}} \frac{1}{\left(\rho+w_{m-1}\right)^{2}} \int_{-\rho}^{w_{m-1}} \frac{\left(\rho+w_{m}\right)^{m-1}}{w_{m}^{m+1}} \int_{0}^{w_{m}} \frac{1}{w_{m+1}^{2}} \cdots \\
& \cdot \int_{0}^{w_{2 m-1}} \frac{1}{w_{2 m-1}^{2}} \int_{0}^{w_{2 m-1}} w_{2 m}^{2 m-1} Y^{(2 m)}\left(w_{2 m}\right) d w_{2 m} \cdots d w_{1}
\end{aligned}
$$

where the integrations are performed along the negative real axis. Since $\left|Y^{(2 m)}(w)\right|$ is a continuous function defined on the compact interval $[-\rho, 0]$, it attains its maximum at some point $w=w_{0},-\rho$ $\leqq w_{0} \leqq 0$. Taking the absolute values and integrating (7), we arrive at

$$
|Y(w)| \leqq\left|Y^{(2 m)}\left(w_{0}\right)\right||w|^{m}|\rho+w|^{m} /(2 m) !, \quad-\rho \leqq w \leqq 0 .
$$

Finally, from (5) and (8), we deduce

$$
\left|Y^{(2 m)}(w)\right| \leqq \frac{1}{(2 m) !}\left|Y^{(2 m)}\left(w_{0}\right)\right|\left|\frac{K\left(1-|\alpha|^{2}\right)}{(1-\bar{\alpha} w)^{2}}\right|^{2 m}|q(w)||w|^{m}|\rho+w|^{m}
$$

for $-\rho \leqq w \leqq 0$; in particular, for $w=w_{0}$,

$$
\begin{aligned}
1 & \leqq \frac{1}{(2 m) !}\left|\frac{K\left(1-|\alpha|^{2}\right)}{\left(1-\bar{\alpha} w_{0}\right)^{2}}\right|^{2 m}\left|q\left(w_{0}\right)\right|\left|w_{0}\right|^{m}\left|\rho+w_{0}\right|^{m} \\
& <\frac{1}{(2 m) !}\left|\frac{K\left(1-|\alpha|^{2}\right)}{\left(1-\bar{\alpha} w_{0}\right)^{2}}\right|^{2 m}\left|q\left(w_{0}\right)\right|\left(1-\left|w_{0}\right|^{2}\right)^{m},
\end{aligned}
$$

contrary to (6). This contradiction proves the theorem.

This theorem for the case $m=2$ was previously obtained by Hadass [2]. 
Disconjugacy criteria of a somewhat different nature may be obtained with the help of the following inequalities [4], [6]: If $p$ is analytic in $D=\{z:|z|<1\}, z=x+i y$, then

$$
|p(w)| \leqq \frac{\int_{0}^{2 \pi}\left|p\left(e^{i \theta}\right)\right| d \theta}{2 \pi\left(1-|w|^{2}\right)}, \quad w \in D,
$$

and

$$
|p(w)| \leqq \frac{\iint_{|z|<1}|p(z)| d x d y}{\pi\left(1-|w|^{2}\right)^{2}}, \quad w \in D .
$$

From these inequalities and Theorem I results the following theorem.

TheOrem II. Let $p$ be analytic in $D=\{z:|z|<1\}$. If

$$
\int_{0}^{2 \pi}\left|p\left(e^{i \theta}\right)\right| d \theta \leqq 2 \pi(2 m) !
$$

or if $m \geqq 2$ and if

$$
\iint_{|z|<1}|p(z)| d x d y \leqq \pi(2 m) !
$$

then Equation (3) is disconjugate in the sense of Reid in $D$.

\section{REFERENCES}

1. G. A. Bessmertnyh and A. Yu. Levin, Some inequalities satisfied by differentiable functions of one variable, Dokl. Akad. Nauk SSSR 144 (1962), 471-474=Soviet Math. Dokl. 3 (1962), 737-740.

2. R. Hadass, On the zeros of the solutions of the differential equation $y^{(n)}(z)+p(z) y(z)$ $=0$, Pacific J. Math. (to appear).

3. W. J. Kim, On the zeros of solutions of $y^{(n)}+p y=0$, J. Math. Anal. Appl. 25 (1969), 189-208.

4. D. London, On the zeros of the solutions of $w^{\prime \prime}(z)+p(z) w(z)=0$, Pacific J. Math. 12 (1962), 979-991.

5. Z. Nehari, The Schwarzian derivative and schlicht functions, Bull. Amer. Math. Soc. 55 (1949), 545-551.

6. - Conformal mapping, 1st ed., McGraw-Hill, New York, 1952, p. 127.

7. - Some criteria of univalence, Proc. Amer. Math. Soc. 5 (1954), 700-704.

8. V. V. Pokornyi, On some sufficient conditions for univalence, Dokl. Akad. Nauk SSSR 79 (1951), 743-746.

9. W. T. Reid, Oscillation criteria for self-adjoint differential systems, Trans. Amer. Math. Soc. 101 (1961), 91-106.

State University of New York, Stony Brook 